\title{
ON THE DERIVATIVE CONES OF POLYHEDRAL CONES
}

\author{
RAMAN SANYAL
}

\begin{abstract}
Hyperbolic polynomials elegantly encode a rich class of convex cones that includes polyhedral and spectrahedral cones. Hyperbolic polynomials are closed under taking polars and the corresponding cones - the derivative cones - yield relaxations for the associated optimization problem and exhibit interesting facial properties. While it is unknown if every hyperbolicity cone is a section of the positive semidefinite cone, it is natural to ask whether spectrahedral cones are closed under taking derivative cones. In this note we give an affirmative answer for polyhedral cones by exhibiting an explicit spectrahedral representation for the first derivative cone. We also prove that higher polars do not have an determinantal representation which shows that the problem for general spectrahedral cones is considerably more difficult.
\end{abstract}

\section{INTRODUCTION}

A homogeneous polynomial $p(\mathbf{x}) \in \mathbb{R}\left[x_{1}, \ldots, x_{n}\right]$ is hyperbolic with respect to a direction $\mathbf{e} \in \mathbb{R}^{n}$ if $p(\mathbf{e})>0$ and for every $\mathbf{q} \in \mathbb{R}^{n}$ the univariate polynomial

$$
\lambda \mapsto p(\mathbf{q}+\lambda \mathbf{e})
$$

has only real roots. The study of hyperbolic polynomials originated in the theory of (hyperbolic) differential equations where it was noted by Gårding [2] that the connected component of $\mathbb{R}^{n} \backslash V(p)$ containing $\mathbf{e}$ is an (open) convex cone. Its closure is called the hyperbolicity cone $\Lambda_{p, \mathbf{e}}$ associated to the pair $(p, \mathbf{e})$. Güler [3] introduced hyperbolic polynomials to convex optimization and showed that interior-point methods can be effectively employed for hyperbolicity cones. More precisely, he showed that $-\log p(\mathbf{x})$ serves as a self-concordant barrier function for $\Lambda_{p, \mathbf{e}}$.

Two of the most important subclasses of hyperbolicity cones are the polyhedral and the spectrahedral cones. For a full-dimensional polyhedral cone

$$
P=\left\{\mathbf{x} \in \mathbb{R}^{n}: \ell_{i}(\mathbf{x}) \geq 0 \text { for all } i=1, \ldots, d\right\},
$$

presented as the intersection of finitely many halfspaces given by linear forms $\ell_{1}(\mathbf{x}), \ldots, \ell_{d}(\mathbf{x})$ it is clear that the polynomial

$$
p(\mathbf{x})=\ell_{1}(\mathbf{x}) \cdot \ell_{2}(\mathbf{x}) \cdots \ell_{d}(\mathbf{x})
$$

is hyperbolic with respect to $\mathbf{e} \in P$ in the interior of $P$ and $\Lambda_{p, \mathbf{e}}=P$. A spectrahedral cone $S$ is the intersection of the cone of positive semidefinite matrices with a linear subspace [11. By choosing a basis for the linear subspace, the cone can be presented by

$$
S=\left\{\mathbf{x} \in \mathbb{R}^{n}: A(\mathbf{x})=x_{1} A_{1}+x_{2} A_{2}+\cdots+x_{n} A_{n} \succeq 0\right\}
$$

Date: October 26, 2018.

2000 Mathematics Subject Classification. 90C22, 52A41, 52B99.

Key words and phrases. polyhedral cones, hyperbolic polynomials, derivative cones, spectrahedra, elementary symmetric functions, polymatroids.

This research was supported by a Miller Research Fellowship at UC Berkeley. 
where $A_{1}, \ldots, A_{n} \in \mathbb{R}^{d \times d}$ are symmetric matrices and $A(\mathbf{x}) \succeq 0$ denotes positive semidefiniteness. In particular, $S$ has a presentation such that $A(\mathbf{e})$ is positive definite for all interior points $\mathbf{e} \in S$. The corresponding hyperbolic polynomial is given by

$$
p(\mathbf{x})=\operatorname{det} A(\mathbf{x})=\operatorname{det} x_{1} A_{1}+x_{2} A_{2}+\cdots+x_{n} A_{n}
$$

and it is easy to see that $p(\mathbf{q}-\lambda \mathbf{e})$ is the characteristic polynomial of a symmetric matrix and hence has only real zeros. These two examples in particular show the richness of the class of convex cones given by hyperbolic polynomials. However, it is still unknown whether this class of convex cones is strictly larger than spectrahedral cones. In a stronger form Peter Lax [7] conjectured that every ternary hyperbolic polynomial has a determinantal representation in the sense of 22 with $A(\mathbf{e})$ positive definite. This conjecture was answered in the positive by Lewis et al. [8] using results of Helton and Vinnikov [5]. In higher dimensions the Lax conjecture is generally false as can be inferred from a count of parameters: hyperbolic polynomials constitute a set with non-empty interior inside the space of forms of fixed degree [9]. In contrast, symmetric matrices of order $d$ yield determinantal forms of type (2) that are constrained to a proper algebraic subset of the space of homogeneous polynomials of degree $d$.

Spectrahedral cones constitute a very nice class of cones from a convex geometric point of view. For instance, spectrahedral cones are facially exposed. This holds true for general hyperbolicity cones as was shown by Renegar [12]. In his investigation of the boundary structure, he considered cones obtained from polarizations of $p(\mathbf{x})$ which yield relaxations of $\Lambda_{p, \mathbf{e}}$ while sharing parts of the facial structure with the original cone. For a homogeneous polynomial $p(\mathbf{x})$, the $i$-th polar with respect to $\mathbf{e}$ is

$$
R_{\mathbf{e}}^{i} p(\mathbf{x}):=\sum_{|\alpha|=i} \mathbf{e}^{\alpha} \frac{\partial^{i}}{\partial \mathbf{x}^{\alpha}} p(\mathbf{x}),
$$

that is, the degree $i$ part in a Taylor expansion of $p(\mathbf{x})$ around $\mathbf{e}$. In particular, the first polar is given by $R_{\mathbf{e}}^{1} p(\mathbf{x})=\sum_{i} e_{i} \frac{\partial}{\partial x_{i}} p(\mathbf{x})=\langle\nabla p(\mathbf{x}), \mathbf{e}\rangle$ and it follows from Rolle's theorem that $R_{\mathbf{e}}^{i} p$ is hyperbolic with respect to $\mathbf{e}$ whenever $p(\mathbf{x})$ is. The corresponding cones are the derivative cones $\Lambda_{p, \mathbf{e}}^{i}=\Lambda_{R_{\mathrm{e}}^{i}, \mathbf{e}}$ of $(p, \mathbf{e})$.

Towards a deeper understanding of the geometry of hyperbolicity cones, it is natural to ask if the subclass of spectrahedral cones is closed under taking derivative cones. The main result of this note is that this is true for the first derivative cone of a polyhedral cone.

Theorem 1.1. Let $P=\left\{\mathbf{x} \in \mathbb{R}^{n}: \ell_{i}(\mathbf{x}) \geq 0\right.$ for $\left.i \in[d]\right\}$ be a full-dimensional polyhedral cone. Let $\mathbf{e} \in \operatorname{int} P$ and assume that $\ell_{i}(\mathbf{e})=1$ for all $i$. Then the first derivative cone is given by all $\mathbf{x} \in \mathbb{R}^{n}$ satisfying

$$
\left[\begin{array}{cccc}
\ell_{1}(\mathbf{x})+\ell_{n}(\mathbf{x}) & \ell_{n}(\mathbf{x}) & \cdots & \ell_{n}(\mathbf{x}) \\
\ell_{n}(\mathbf{x}) & \ell_{2}(\mathbf{x})+\ell_{n}(\mathbf{x}) & \cdots & \ell_{n}(\mathbf{x}) \\
\vdots & \vdots & \ddots & \vdots \\
\ell_{n}(\mathbf{x}) & \ell_{n}(\mathbf{x}) & \cdots & \ell_{n-1}(\mathbf{x})+\ell_{n}(\mathbf{x})
\end{array}\right] \succeq 0 .
$$

Let us illustrate the theorem with an example that motivated this research.

Example 1.2. The 3-dimensional halfcube $H_{3}$ is the subpolytope of the 3 -cube $[-1,1]^{3}$ induced by the vertices with an even number of -1 coordinates. It is the affine slice of the polyhedral cone below with $\{t=1\}$. The corresponding polynomial $p$ is hyperbolic with respect to $\mathbf{e}=(1,0,0,0)$. The same affine section of its first derivative cone is the 3-dimensional elliptope [6] (also known as the Samosa). 

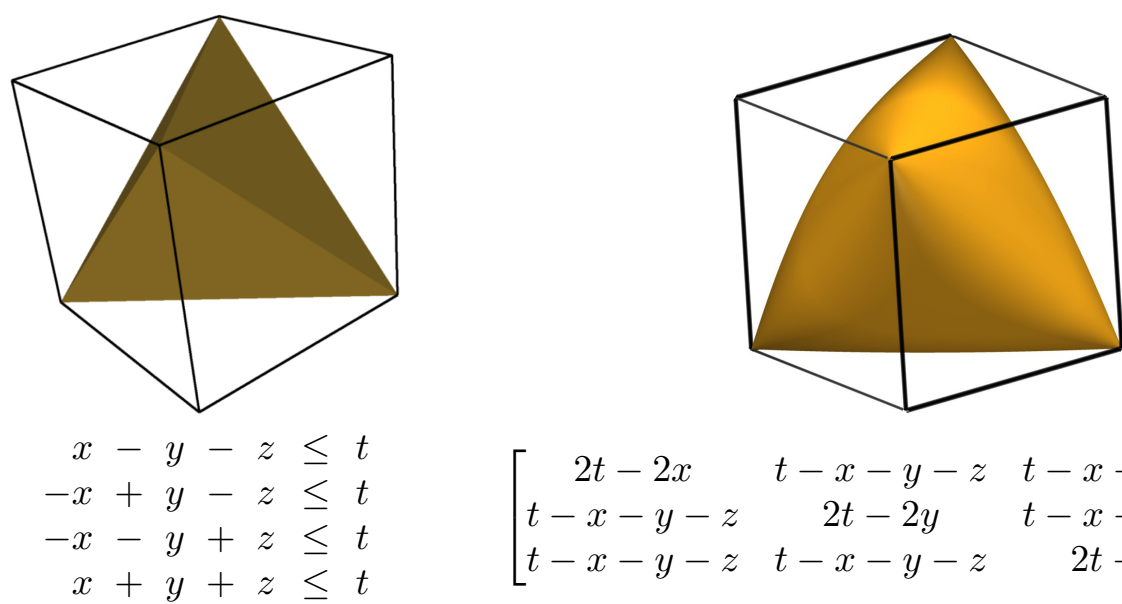

$$
\left[\begin{array}{ccc}
2 t-2 x & t-x-y-z & t-x-y-z \\
t-x-y-z & 2 t-2 y & t-x-y-z \\
t-x-y-z & t-x-y-z & 2 t-2 z
\end{array}\right] \succeq 0
$$

The Samosa has 6 one-dimensional faces corresponding to the edges of the halfcube. The first polar is the equation Cayley cubic surface $t^{3}-t x^{2}-t y^{2}-t z^{2}+2 x y z$. Unfortunately, this nice connection between halfcubes and elliptopes does not hold in higher dimensions.

In fact, the above theorem asserts the much stronger statement that the first polar of a product of linear forms satisfies the generalized Lax conjecture, that is, the first polar of $p(\mathbf{x})=\prod_{i} \ell_{i}(\mathbf{x})$ equals the determinant of the given matrix. However, this is the only polar for which this is true.

Theorem 1.3. Derivative cones of spectrahedra do not satisfy the generalized Lax conjecture.

The prototypical example of a polyhedral cone is given by $\mathbb{R}_{\geq 0}^{d}$ with hyperbolic polynomial $p(\mathbf{x})=$ $E_{d}(\mathbf{x})=x_{1} x_{2} \cdots x_{d}$, the $d$-th elementary symmetric function. In particular, $E_{d}(\mathbf{x})$ is hyperbolic with respect to $\mathbf{e}=\mathbf{1}$ and $R_{\mathbf{1}}^{i} E_{d}(\mathbf{x})=E_{d-i}(\mathbf{x})$ are elementary symmetric functions. The relation of the corresponding cones to semidefinite programming was considered in Zinchenko [13] who showed that $\Lambda_{p, \mathbf{e}}^{i}$ is a spectrahedral shadow, i.e. the projection of a spectrahedral cone. However, this is a weaker statement in that spectrahedral cones are not closed under projection and images under projection have much weaker properties such as non-exposed faces. The main focus of [13] was a description for the dual cone to $\Lambda_{E_{n-1}, \mathbf{1}}$ which can be inferred from Theorem 1.1. The remainder of the paper is concerned with the proof of the two theorems above.

Acknowledgments. The author would like to thank the organizers of the AIM workshop "Convex algebraic geometry, optimization and applications" where this question was raised. We would like to thank Thilo Rörig and Philipp Rostalski for fruitful discussions and Bernd Sturmfels for support and encouragement.

\section{Proofs Via REALizations of UNiform MATRoids}

Before we come to the proof of Theorem 1.1, let us reduce the statement to the case of the positive orthant $\mathbb{R}_{\geq 0}^{d}$ with hyperbolic polynomial $E_{d}(\mathbf{x})=x_{1} x_{2} \cdots x_{d}$. To that end, note that for $p(\mathbf{x})=\prod_{i}^{d} \ell_{i}(\mathbf{x})$, the $i$-th polar satisfies

$$
R_{\mathbf{e}}^{i} p(\mathbf{x})=i ! p(\mathbf{e}) E_{d-i}\left(\ell_{1}(\mathbf{x}), \ldots, \ell_{d}(\mathbf{x})\right)
$$

where $E_{d-i}\left(y_{1}, \ldots, y_{d}\right)$ is the $d-i$-th elementary symmetric function. Geometrically, this means that the $i$-th derivative cone of $P=\mathbb{R}_{\geq 0}^{d} \cap L$, where $L$ is a linear subspace, equals the intersection of $L$ with the $i$-th derivative cone of $\mathbb{R}_{>0}^{d}$. The individual linear forms $\ell_{i}(\mathbf{x})$ are called the eigenvalues of hyperbolic polynomial $p(\mathbf{x})$ and the above claim holds true for general hyperbolic polynomials [12, Prop. 18]. 
While Theorem 1.1 can be proved inductively by verifying that the determinant of the shown matrix equals the first polar of $p(\mathbf{x})$ with respect to $\mathbf{1}$, we will make use of a beautiful connection between hyperbolic polynomials and polymatroids due to Gurvits [4] and spectrahedra and realizations of polymatroids due to Choe et al. [1. Recall that a polymatroid on the ground set $[n]=\{1,2, \ldots, n\}$ is a monotone submodular function $\mathrm{rk}: 2^{[n]} \rightarrow \mathbb{Z}_{\geq 0}$. That is, for any $I, J \subseteq[n]$ we have $0 \leq \operatorname{rk}(I) \leq \operatorname{rk}(I \cup J)$ and

$$
\operatorname{rk}(I \cup J) \leq \operatorname{rk}(I)+\operatorname{rk}(J)-\operatorname{rk}(I \cap J) .
$$

If, in addition, $\operatorname{rk}(I) \leq|I|$ for all $I \subseteq[n]$, then rk defines a matroid; see [10, Sect. 12.1] for more information. Matroids and polymatroids are combinatorial abstractions of subspace arrangements: Let $V_{1}, V_{2}, \ldots, V_{n}$ be linear subspaces of a given vector space $V$, then

$$
I \mapsto \operatorname{dim} \sum_{i \in I} V_{i}
$$

yields a polymatroid. The case of matroids corresponds to line arrangements. Polymatroids from subspace arrangements are called realizable polymatroids and it is well known that not all polymatroids are realizable [10, Ch. 6].

Example 2.1 (Uniform matroids). For every $0 \leq k \leq n$, the function $\operatorname{rk}_{k}(I)=\min (k,|I|)$ defines a matroid, called the uniform matroid $U_{k, n}$. A realization of $U_{k, n}$ is given by any generic choice of $n$ lines in a $k$-dimensional vector space.

The connection between hyperbolic polynomials and polymatroids was first observed by Gurvits [4]. For a univariate polynomial $p(t) \in \mathbb{R}[t]$, let mult $_{0}(p(t))$ be the order of vanishing at the origin. For a subset $I \subseteq[n]$, denote by $\chi_{I} \in\{0,1\}^{n}$ its characteristic vector.

Theorem 2.2 ([4, Fact 5.3]). Let $p(\mathbf{x})$ be a d-form hyperbolic with respect to $\mathbf{e}$ and assume that $\mathbb{R}_{\geq 0}^{n} \subseteq \Lambda_{p, \mathbf{e}}$. Then the map $\operatorname{rk}_{p}: 2^{[n]} \rightarrow \mathbb{Z}$ given by

$$
I \mapsto d-\operatorname{mult}_{0}\left(p\left(\chi_{I}+\lambda \mathbf{e}\right)\right)
$$

defines a polymatroid.

It is easy to see that for $e=\mathbf{1}$, the polymatroid associated to the $k$-th elementary symmetric function $E_{k}(\mathbf{x})$ is exactly the uniform matroid $U_{k, n}$.

The connection to spectrahedral cones is that the form (1) yields a realization of the associated polymatroid. To see this, let us assume that the matrix map

$$
A(\mathbf{x})=x_{1} A_{1}+x_{2} A_{2}+\cdots+x_{n} A_{n}
$$

with symmetric $A_{1}, \ldots, A_{n} \in \mathbb{R}^{d \times d}$ satisfies $A(\mathbf{e})=$ Id. If $\operatorname{det} A(\mathbf{x})$ satisfies the condition of Theorem 2.2 then every $A_{i}$ is positive semidefinite and hence is of the form $A_{i}=L_{i} L_{i}^{T}$ for some real matrix $L_{i} \in \mathbb{R}^{d \times k_{i}}$. Now, $p\left(\chi_{I}+\lambda \mathbf{e}\right)=\operatorname{det}\left(A\left(\chi_{I}\right)+\lambda \mathrm{Id}\right)$ is the characteristic polynomial of $A\left(\chi_{I}\right)$. The order of vanishing is the nullity of $L_{I}^{T}$, where $L_{I}=\left(L_{i}: i \in I\right)$ is the concatenation of $L_{i}$ for $i \in I$. Hence $d-\operatorname{det}\left(A\left(\chi_{I}\right)-\lambda \mathrm{Id}\right)$ is the rank of $L_{I}$. The observation of Choe et al. [1] is that the hyperbolic polynomial $p(\mathbf{x})=\operatorname{det}(A(\mathbf{x}))$ contains information about the realization given by $L=\left(L_{1}, L_{2}, \ldots, L_{n}\right)$. In our context, it is sufficient to assume that $L_{i}$ is actually a single vector. Let $X=\operatorname{diag}\left(x_{1}, \ldots, x_{n}\right)$, then

$$
p(\mathbf{x})=\operatorname{det} A(\mathbf{x})=\operatorname{det}\left(L \cdot X \cdot L^{T}\right)=\sum\left\{\operatorname{det}\left(L_{I}\right)^{2} \mathbf{x}^{I}: I \subseteq[n],|I|=d\right\}
$$

where the last equality follows from the Binet-Cauchy theorem.

We are now able to give the proofs of Theorems 1.1 and 1.3. For the former, we need to find a realization of the uniform matroid $U_{n-1, n}$ in the form of a matrix $L \in \mathbb{R}^{(n-1) \times n}$ such that 
every maximal minor is \pm 1 . Such a unimodular realization for $U_{n-1, n}$ is given by $L=(\mathrm{Id}, \mathbf{- 1})$. Theorem 1.3 follows from the well-known fact that $U_{k, n}$ has no unimodular realization unless $k \leq 1$ or $k \geq n-1$. Indeed, if $2 \leq k \leq n-2$ and $L \in \mathbb{R}^{k \times n}$ matrix giving an unimodular realization of $U_{k, n}$ then, without loss of generality, $L=\left(\mathrm{Id}, L^{\prime}\right)$ with $L^{\prime} \in \mathbb{R}^{k \times(n-k)}$. The conditions on the maximal minors of $L$ forces $L^{\prime}$ to have all minors \pm 1 which is only possible iff $L^{\prime}$ has at most one row or column. This proves Theorems 1.1 and 1.3 .

Let us remark that this does not exclude the possibility that the hyperbolicity cones for $E_{k}(\mathbf{x})$ are slices of the PSD cone. Indeed, it would be sufficient to find an polynomial $g(\mathbf{x})$ hyperbolic with respect to $\mathbf{e}=\mathbf{1}$ such that $\Lambda_{E_{k}, \mathbf{e}} \subseteq \Lambda_{g, \mathbf{e}}$ and $g E_{k}$ has a symmetric determinantal representation with $A(\mathbf{e}) \succ 0$. We close by showing this for $E_{2}(\mathbf{x})$. To this end, consider the spectrahedral cone given by

$$
A(\mathbf{x})=\left[\begin{array}{ccccc}
E_{1}(\mathbf{x}) & x_{1} & x_{2} & \cdots & x_{n-1} \\
x_{1} & E_{1}(\mathbf{x}) & & & \\
x_{2} & & E_{1}(\mathbf{x}) & & \\
\vdots & & & \ddots & \\
x_{n-1} & & & & E_{1}(\mathbf{x})
\end{array}\right] \succeq 0 .
$$

It is easy to see that $A(\mathbf{1})$ is positive definite and

$$
\operatorname{det} A(\mathbf{x})=2 E_{1}(\mathbf{x})^{n-2} E_{2}(\mathbf{x}) .
$$

The arrangement of 2-planes is not very well understood. As of now, we do not know spectrahedral representations for all elementary symmetric functions but we conjecture that they exist.

\section{REFERENCES}

[1] Y.-B. Choe, J. G. Oxley, A. D. Sokal, And D. G. Wagner, Homogeneous multivariate polynomials with the half-plane property, Adv. in Appl. Math., 32 (2004), pp. 88-187. Special issue on the Tutte polynomial.

[2] L. Gårding, An inequality for hyperbolic polynomials, J. Math. Mech., 8 (1959), pp. 957-965.

[3] O. GüLER, Hyperbolic polynomials and interior point methods for convex programming, Math. Oper. Res., 22 (1997), pp. $350-377$.

[4] L. GuRvits, Combinatorial and algorithmic aspects of hyperbolic polynomials. preprint, math.C0/0404474, April 2005.

[5] J. W. Helton And V. Vinnikov, Linear matrix inequality representation of sets, Comm. Pure Appl. Math., 60 (2007), pp. 654-674.

[6] M. Laurent And S. Poljak, On the facial structure of the set of correlation matrices, SIAM J. Matrix Anal. Appl., 17 (1996), pp. 530-547.

[7] P. D. LAX, Differential equations, difference equations and matrix theory, Comm. Pure Appl. Math., 11 (1958), pp. 175-194.

[8] A. S. Lewis, P. A. Parrilo, and M. V. Ramana, The Lax conjecture is true, Proc. Amer. Math. Soc., 133 (2005), pp. 2495-2499 (electronic).

[9] W. NuIJ, A note on hyperbolic polynomials, Math. Scand., 23 (1968), pp. 69-72 (1969).

[10] J. G. OxLey, Matroid theory, Oxford Science Publications, The Clarendon Press Oxford University Press, New York, 1992.

[11] M. Ramana And A. J. Goldman, Some geometric results in semidefinite programming, J. Global Optim., 7 (1995), pp. 33-50.

[12] J. Renegar, Hyperbolic programs, and their derivative relaxations, Found. Comput. Math., 6 (2006), pp. 5979.

[13] Y. Zinchenko, On hyperbolicity cones associated with elementary symmetric polynomials, Optim. Lett., 2 (2008), pp. 389-402.

Raman Sanyal, Department of Mathematics, FU Berlin, Arnimallee 2, 14195 Berlin, Germany

E-mail address: sanyal@math.fu-berlin.de 